\title{
Assessment of Malt Barley Genotypes for Grain Yield and Malting Quality Traits in the Central Highlands of Ethiopia
}

\author{
Thomas Tsige $^{1} \quad$ Tigist Shiferaw $^{1} \quad$ Shimles Gezahegn $^{2} \quad$ Kefyalew Taye $^{3}$ \\ 1.Holetta Agricultural Research Center, P.O. Box 31, Holetta, Ethiopia \\ 2.Kulumsa Agricultural Research Center, P.O. Box 489, Assela, Ethiopia \\ 3.Debreberhan Agricultural Research Center, P.O. Box 112, Debreberhan, Ethiopia
}

\begin{abstract}
A multi-location variety trial was conducted with the objective to identify suitable malt barley varieties that satisfy the malt and brewing industry quality requirements and reduce the cost for importing malt barley. The trial was conducted to evaluate the performance of 28 promising malt barley varieties in seven environments (site-season combinations) during 2017 and 2018 cropping seasons. The phenological and agronomic data collected were subjected to analysis of variance (ANOVA) using ' $\mathrm{R}$ ' software. All the traits analysis showed significant genotype, environment and genotype by environment interaction effects. Genotype, HB 52 X Bahati (G-5) exhibited high mean grain yield $\left(5128 \mathrm{kgha}^{-1}\right)$ and significantly different for grain yield from one of the improved checks (Traveller). The other promising genotype, Bekoji-1 X Grace (G-8) showed acceptable malt quality results for extract $(81.8 \%)$, protein $(10.0 \%)$, friability $(85 \%)$ and lower values for beta -glucan $(369 \mathrm{mg} / \mathrm{l})$ with comparable grain yield to the improved checks (Traveller, HB1963). Similarly, genotype Sabin X Beka (G-7) showed acceptable malt quality results with lower level of beta-glucan $(287 \mathrm{mg} / \mathrm{l})$. The "which-won-where" and "Mean vs. Stability" view of GGE biplot showed that, G-5 exhibited high mean grain yield and moderate grain yield stability and is the winner genotype in all test environments. Overall, G-8 is identified as potential malting barley candidate varieties to be tested for more industrial malt quality traits prior to variety verification trial and G-5 can be considered as potential parent in the malt barley crossing program for its good yield potential.
\end{abstract}

Keywords: ANOVA, GGE biplot, Malt barley, Grain yield, Malt quality and Stability

DOI: $10.7176 / \mathrm{JBAH} / 10-20-01$

Publication date:October $31^{\text {st }} 2020$

\section{INTRODUCTION}

Barley is one of the most important cereal crops widely grown in the highlands of Ethiopia with annual production of about 2.0 million tons cultivated on an area of about 1 million hectares of land with an average national yield of 2.16 tons/ha (CSA, 2018). In the barley-based farming systems of the central highlands, smallholder farmers have very few alternative crops. One source of income could be growing malting barley, which has dependable local buyers in the country (Bayeh and Berhane, 2011). Both food and malt barley are grown side by side sharing similar agro-ecologies. However, the share of malt barley is roughly $15-20 \%$ of the total barley production, which is the major input for beer production (Berhane et al., 2016). Traditionally, both six-row and two row barley types are cultivated in the country, but the best malt quality for beer is produced from two row varieties.

Malt barley is a high-opportunity cash crop, with great room for profitable expansion, particularly when connected to growing breweries. However, there is a shortage of quality malt barley varieties to meet the demand of the local breweries that forced the malt factories to import large quantity of malt barley from abroad. The gap between domestic supply and demand indicates an opportunity to enhance local production and substitute import through huge untapped malt barley potential in the country. Malt imports has grown tremendously reaching over 75 thousand tons in 2017 covering about $70 \%$ of total annual demand and costing the country about 41.5 million USD (ERCA, 2017).

Malt barley production has not expanded as expected, despite the potential of the country to grow malting barley both in quality and quantity. There is a relatively huge domestic market for malt of reasonable quality, where large number of farmers in the highlands of Ethiopia can commit part of their barley area to malt barley production. Even though barley grows in many highland regions of the country, the adoption of the malt barley vartieties is limited to the Arsi highlands and to a lessor extent in Bale where farmers can sale their proudce to the Asela malt factory and to the emerging brewery companies such as Heniken, Diageo and Dashen. The lack of adoption of malt barley to other highland barley producing areas is due to limited extension activities by the ministry of agriculture and relevant organizations. Curently, there is an attempt to promote malt barley production in the central highlands of Oromia and Amhra region to provide malt to breweries through contractual production. Therefore, improving the knowledge and skill of farmers through demonstrating new malt barley varieties would be vital to increase production and productivity to fill the existing supply gap in the country. In addition the quality demand from the brweries and the malt factory is a bench mark for the malt barley breeding program. However, most of the nationally released malt barley varieties did not satisfy all the requierments that showed the importance of developing breeding activity to release the malt barley varieties that satisfy the quality parameter demand of the 
brwereies and the malt factories.

The National Agricultural Research system (NARS), has developed more than 15 malt barley varieties over the last three decades with the collaboration of national research system and international organizations. This paper presents the results of the malt barley multi-location variety trial conducted in seven environments. The target was to select high yielding, agronomically superior varieties with good malting quality traits, disease and pest resistance and promot best performing varieties for release or incorporate in the breeding pipeline development as recipients/donors of useful genes.

\section{MATERIALS AND METHODS}

Twenty eight malt barley genotypes were evaluated in two sets of experiment in 2017 and 2018 cropping seasons and from these genotypes twelve of them were evaluated repeatedly in both years. However, the remaining sixteen genotypes appeared once in each year (Table 1). Therefore, twenty materials were tested each year using RCBD design with three replications. The experiments were carried out at Holetta $\left(9^{\circ} 00^{\prime} \mathrm{N}, 38^{\circ} 38^{\prime} \mathrm{E}\right.$, elevation $\left.2400 \mathrm{~m}\right)$, Bekoji $\left(7^{\circ} 15^{\prime} \mathrm{N}, 39^{\circ} 15^{\prime} \mathrm{E}\right.$, elevation $\left.2830 \mathrm{~m}\right)$, Kofele $\left(7^{\circ} 00^{\prime} \mathrm{N}, 38^{\circ} 45^{\prime} \mathrm{E}\right.$, elevation $\left.2700 \mathrm{~m}\right)$ and Debrebrhane $\left(9^{\circ} 41^{\prime} \mathrm{N}, 39^{\circ} 32^{\prime} \mathrm{E}\right.$, elevation $2800 \mathrm{~m}$ ) experimental sites in a non-orthogonal set of six environments (site-season combinations). Twenty two of the experimental materials were selected from 2016 and 2017 malt barley preliminary variety trials and the other six genotypes were included as checks (Table 1).

Data were recorded on the following phenological and agronomic traits: days to $50 \%$ heading, days to $50 \%$ maturity, plant height $(\mathrm{cm})$, hectoliter weigh $\left(\mathrm{Kg} \mathrm{hl}^{-1}\right)$, thousand kernel weight $(\mathrm{gm})$ and grain yield $\left(\mathrm{Kg} \mathrm{ha}^{-1}\right)$ from four central rows. Plot yields were adjusted to $12.5 \%$ moisture content and converted to kilogram per hectare. Disease data were recorded on scald and net blotch on $0-9$ scale and changed to percentage data, where $0=0 \%$, $1=3 \%, 2=12 \%, 3=25 \%, 4=42 \%, \quad 5=58 \%, 6=75 \%, 7=88 \%, 8=97 \%, 9=100 \%$ before transformed using angular transformation for statistical analysis. These traits were subjected to analysis of variance using R- software (R Core Team, 2017). In the analysis the environments were considered as random and genotypes as fixed effects, and a mixed effect model ANOVA was used for statistical analysis. The individual and combine environment analysis of variance of the experiment was conducted as the model suggested by Singh and Ceccarelli (1996).

$\mathrm{Y}_{\mathrm{ij}}=\mu+\mathrm{G}_{\mathrm{i}}+\mathrm{B}_{\mathrm{j}}+\mathrm{e}_{\mathrm{ij}}$ and $\mathrm{Y}_{\mathrm{ijk}}=\mu+\mathrm{G}_{\mathrm{i}}+\mathrm{E}_{\mathrm{j}}+\mathrm{GE}_{\mathrm{ij}}+\mathrm{Bk}_{(\mathrm{j})}+\mathrm{e}_{\mathrm{ijk}}$.

Where, $Y_{i j}=$ observed value of genotype $i$ in block $j, Y_{i j k}=$ observed value of genotype $i$ in block $k$ of environment $j, \mu=$ grand mean of the experiment, $G_{i}=$ the effect of genotype $i, B_{j}=$ the effect of block $j, B k_{(j)}=$ the effect of block $\mathrm{k}$ in environment $\mathrm{j}, \mathrm{e}_{\mathrm{ij}}=$ error effect of genotype $\mathrm{i}$ in block $\mathrm{j}, \mathrm{E}_{\mathrm{j}}=$ environment effect, $\mathrm{GE}_{\mathrm{ij}}=$ the interaction effect of genotype $\mathrm{i}$ with environment $\mathrm{j}, \mathrm{e}_{\mathrm{ijk}}=$ error (residual) effect of genotype $\mathrm{i}$ in block $\mathrm{k}$ of environment $\mathrm{j}$. GGE bi-plots were performed on grain yield to determine stability of the genotypes using GGEBiplotGUI packages of R- software (R Core Team, 2017).

The malt quality traits, namely extract content $[\% \mathrm{DM}]$, protein content $[\% \mathrm{DM}]$, friability $[\%]$ and $\beta$-glucan content $[\mathrm{mg} / \mathrm{L}]$ of selected genotypes were analyzed using the wet chemistry method in Germany malt quality laboratory "Versuchs- und Lehranstalt für Brauerei in Berlin" on malted grain following the appropriate procedure. Malt extract content was determined according to a small-scale version of the European Brewery Convention (EBC) Methods Manual, Section 4.9.1 (European Brewery Convention, 1998). Additionally, grain samples of all genotypes included in this study were analysed at Holetta quality laboratory following Near infrared spectroscopy (NIRs) technique using Bruker Tango instrument.

Table 1. Lists of malt barley genotypes and environments used for the trials

\begin{tabular}{lllllllll}
\hline Trt & Genotype & Year & Trt & Genotype & Year & Loc & Year & Env \\
\hline G1 & Grace x HB 1307 & $17-18$ & G15 & KWS_Grinada & 2017 & Holetta & 2017 & HO17 \\
G2 & Bekoji I xBahati & $17-18$ & G16 & KWS-Hobbs & 2017 & Bekoji & 2017 & BK17 \\
G3 & HB 1307 x Su-Lilly & $17-18$ & G17 & KWS-Sassy & 2017 & Bekoji & 2018 & BK18 \\
G4 & Belgium 2 & $17-18$ & G18 & KWS_Canton & 2017 & Kofele & 2017 & KF17 \\
G5 & HB 52 x Bahati & $17-18$ & G19 & KWS-Solicit & 2017 & Kofele & 2018 & KF18 \\
G6 & IBON 174/03 x Traveller & $17-18$ & G20 & IBON-HI13/14-41 & 2018 & D/berhane & 2018 & DB18 \\
G7 & Sabini x Beka & $17-18$ & G21 & IBON-HI14/15-45 & 2018 & & & \\
G8 & Bekoji-1 x Grace & $17-18$ & G22 & IBON-HI14/15-56 & 2018 & & & \\
G9 & IBON 174/03* & $17-18$ & G23 & IBON-HI14/15-96 & 2018 & & & \\
G10 & Holker & $17-18$ & G24 & IBON-HI14/15-102 & 2018 & & & \\
G11 & HB 1963 * & $17-18$ & G25 & IBON-HI14/15-147 & 2018 & & & \\
G12 & HB 1964 & $17-18$ & G26 & MBHIBYT-23 & 2018 & & & \\
G13 & KWS-Dante & 2017 & G27 & Explorer & 2017 & & & \\
G14 & KWS-Eileen & 2017 & G28 & Traveller $* *$ & 2018 & & & \\
\hline
\end{tabular}

*improved Check, locally developed; ** = Improved check, Introduced, G1-G12 evaluated for two years, G13G28 evaluated for one year 


\section{RESULTS AND DISCUSSION}

The combined analysis of variance, across seven environments showed highly significant variations among the malt barley genotypes tested for all quantitative traits considered. The differences among the environments were statistically significant for all traits. Similarly, genotype by environment interaction was significant for all traits (Table 2). It showed that all genotypes were not affected by environment equally. This G x E interaction effect made the selection process difficult and requires further stability analysis of the genotype to select the one relatively stable across environments and provide high yield.

Table 2. Mean squares of traits of 28 malt barley genotypes grown at six environments

\begin{tabular}{llllllllll}
\hline & DF & DHE & DMA & PLH & SC(DF) $\S$ & NB(DF) $\S$ & TKW & HLW & GYLD \\
\hline Gen & 27 & $709^{* *}$ & $461^{* *}$ & $4370^{* *}$ & $1719(27)^{* *}$ & $572(27)^{* *}$ & $295^{* *}$ & $35^{* *}$ & $11779569^{* *}$ \\
Env & 5 & $2599^{* *}$ & $4063^{* *}$ & $4501^{* *}$ & $9367(4)^{* *}$ & $12017(4)^{* *}$ & $1726^{* *}$ & $826^{* *}$ & $61902099^{* *}$ \\
Gen:env & 87 & $26^{* *}$ & $53^{* *}$ & $131^{* *}$ & $214(68)^{* *}$ & $254(68)^{* *}$ & $17^{* *}$ & $7^{* *}$ & $932567^{* *}$ \\
Env:rep & 12 & $21^{* *}$ & $16 \mathrm{~ns}$ & $107^{* *}$ & $188(10)^{* *}$ & $61(10)$ & $9 \mathrm{~ns}$ & $1 \mathrm{~ns}$ & $2828458^{* *}$ \\
Residuals & 228 & 9 & 16 & 40 & $74(190)$ & $67(190)$ & 8 & 4 & 441982 \\
\hline CV & & 3.56 & 2.78 & 6.61 & 16.35 & 39.31 & 5.82 & 2.94 & 16.92 \\
Mean & & 84.6 & 143.4 & 96.0 & 52.5 & 20.9 & 47.5 & 67.6 & 3929.7 \\
\hline
\end{tabular}

$\mathrm{DF}=$ degree of freedom, $\mathrm{DHE}=$ days to heading, $\mathrm{DMA}=$ days to maturity, $\mathrm{PLH}=$ plant height $(\mathrm{cm})$,

$\mathrm{SC}=$ scald $(\%), \mathrm{NB}=$ net blotch $(\%), \mathrm{TKW}=$ thousand kernel weight $(\mathrm{g}), \mathrm{HLW}=$ hectoliter weight

$\left(\mathrm{Kghl}^{-1}\right), \mathrm{GYLD}=$ grain yield $\left(\mathrm{kg} \mathrm{ha}^{-1}\right), * *, *$ significant at $5 \%$ and $1 \%$ probability level, $\mathrm{ns}=$ non

significant, §these traits were not recorded at DB18 and mean squares under those traits

are angular transformed values

Among the tested genotypes even though not significantly different from some of the newly tested genotypes and check varieties (IBON 174/03, HB 1963, HB 1964), G5 (HB 52 x Bahati) showed highest mean grain yield. However, it had significantly highest mean grain yield than the registered European varieties (Explorer and Traveller). Then most genotypes (G21-G26) that were substituted during 2018 cropping season had higher mean grain yield comparable to G5, but it should be noticed that these genotypes were evaluated only at three environments (BK18, KF18 and DB18). Similarly, among the test genotypes evaluated at all environments, G6 (IBON 174/03 x Traveller) scored better mean grain yield. Whereas, the introduced malt barley (G13-G19) materials scored the lowest mean grain yield values. The maximum hectoliter weight (HLW) were recorded for the check variety G11 (HB1963), genotype G8 (Bekoji-1 x Grace) and G26 (MBHIBYT-23). Significantly higher mean TKW value was recorded on HB1964 (56.6g) and KWS-Eileen (52.6g). On the other hand, most introduced malt barley genotypes (viz. G13, G14, G15 G16, G17 and G19) had higher mean scald value of 67, 65, 69, 63, 67 and $72 \%$, respectively. This is due to the fact that these materials evaluated under different environment condition than Ethiopia (Table 3). However, materials derived from crossing program had relatively better tolerance to scald. Accordingly, G1, G3, G4, G5, G6, G7 and G8 scored mean scald value of 53, 46, 45, 38, 51, 48 and $54 \%$. Regarding net blotch most of tested materials showed moderate resistant. In contrast, these genotypes which had higher scald values showed lower net blotch scores. As an example G15, G22 and G27 scored 69, 80 and 74\% for scald and 9,23 and 10\% for net blotch, respectively. This may be due to the confounding effect of scald on net blotch. Plant height showed consistently large variation among the malt barley varieties. Similarly, most foreign materials have short plant height, in contrast G7 revealed high mean plant height value of $116 \mathrm{~cm}$ followed by G8 (115 cm). G6 (IBON 174/03 x Traveller) and G9 (IBON 174/03) were relatively early whereas G-17 (KWS-Sassy), G14 (KWS-Eileen), G-18 (KWS-Canton) and other European introduced materials were late in days to maturity. Generally, among the malt barley genotypes tested in all environments, HB $52 \mathrm{x}$ Bahati and IBON 174/03 x Traveller showed grain yield advantage as compared to the recently released check varieties (HB1963 and HB 1964) and better disease resistance (Table 3). Similarly, Bekoji-1 x Grace had comparable mean grain yield value as the standard checks (HB 1963, Traveller) and high values in mean grain physical quality parameters (TKW and HLW). In addition, the newly inserted genotypes (G21-G26) showed similar grain yield with recent check varieties and to confirm their performance over year, these genotypes genotypes will be evaluated again in 2019/20 cropping season. 
Table 3. Over all mean for eight traits of 28 malt barley genotypes tested during the 2017 and 2018 main cropping season

\begin{tabular}{|c|c|c|c|c|c|c|c|c|c|c|}
\hline Trt\# & Genotype & & DHE & DMA & PLH & SC§ & NB§ & TKW & HLW & GYLD \\
\hline G1 & Grace x HB 1307 & & $84^{\mathrm{c}-\mathrm{g}}$ & $143^{d-j}$ & $114^{\mathrm{ab}}$ & $53^{\mathrm{d}-\mathrm{i}}$ & $35^{\mathrm{abc}}$ & $49.5^{\mathrm{b}-\mathrm{g}}$ & $69.8^{\mathrm{ab}}$ & $3995^{\mathrm{cd}}$ \\
\hline G2 & Bekoji I xBahati & & $79^{i-1}$ & $137^{\mathrm{jk}}$ & $97^{\text {fgh }}$ & $71^{\mathrm{abc}}$ & $25^{\mathrm{b}-\mathrm{g}}$ & $44.1^{\mathrm{hi}}$ & $68.3^{\mathrm{a}-\mathrm{e}}$ & $3972^{\mathrm{cd}}$ \\
\hline G3 & HB 1307 x Su-Lilly & & $84^{\mathrm{c}-\mathrm{g}}$ & $139^{\mathrm{h}-\mathrm{k}}$ & $110^{\mathrm{a}-\mathrm{d}}$ & $46^{\mathrm{g}-\mathrm{j}}$ & $30^{\mathrm{b}-\mathrm{e}}$ & $48.9^{\mathrm{b}-\mathrm{g}}$ & $68.3^{\mathrm{a}-\mathrm{e}}$ & $3920^{\text {cde }}$ \\
\hline G4 & Belgium 2 & & $84^{\mathrm{d}-\mathrm{h}}$ & $138^{\mathrm{ijk}}$ & $111^{\mathrm{abc}}$ & $45^{\text {hij }}$ & $31^{\text {bcd }}$ & $47.7^{\mathrm{d}-\mathrm{g}}$ & $68.9^{\mathrm{a}-\mathrm{d}}$ & $4049^{\text {bcd }}$ \\
\hline G5 & HB $52 \times$ Bahati & & $81^{\mathrm{g}-\mathrm{j}}$ & $141^{\mathrm{f}-\mathrm{k}}$ & $106^{\mathrm{b}-\mathrm{e}}$ & $38^{\mathrm{ij}}$ & $27^{\mathrm{b}-\mathrm{f}}$ & $46.6^{\mathrm{ghi}}$ & $64.0^{\mathrm{g}}$ & $5128^{a}$ \\
\hline G6 & $\begin{array}{ll}\text { IBON } & 174 / 03 \\
\text { Traveller } & \end{array}$ & $\mathrm{x}$ & $73^{1}$ & $136^{\mathrm{k}}$ & $96^{\mathrm{gh}}$ & $51^{e-j}$ & $23^{\mathrm{c}-\mathrm{g}}$ & $51.2^{\mathrm{bc}}$ & $67.6^{\mathrm{b}-\mathrm{e}}$ & $4470^{\mathrm{a}-\mathrm{d}}$ \\
\hline G7 & Sabini x Beka & & $82^{\mathrm{e}-\mathrm{i}}$ & $140^{\mathrm{f}-\mathrm{k}}$ & $116^{\mathrm{a}}$ & $48^{\mathrm{f}-\mathrm{j}}$ & $31^{\mathrm{b}-\mathrm{e}}$ & $50.7^{\mathrm{b}-\mathrm{e}}$ & $68.6^{\mathrm{a}-\mathrm{d}}$ & $3931^{\text {cde }}$ \\
\hline G8 & Bekoji-1 x Grace & & $81^{\mathrm{g}-\mathrm{j}}$ & $140^{\mathrm{f}-\mathrm{k}}$ & $115^{\mathrm{ab}}$ & $54^{\mathrm{c}-\mathrm{i}}$ & $29^{\mathrm{b}-\mathrm{e}}$ & $51.0^{\mathrm{b}-\mathrm{d}}$ & $70.1^{\mathrm{ab}}$ & $4027^{\text {bcd }}$ \\
\hline G9 & IBON $174 / 03^{*}$ & & $75^{\mathrm{kl}}$ & $135^{\mathrm{k}}$ & $88^{\mathrm{h}}$ & $54^{\mathrm{c}-\mathrm{i}}$ & $28^{\mathrm{b}-\mathrm{e}}$ & $48.8^{\mathrm{b}-\mathrm{g}}$ & $67.0^{\text {cde }}$ & $4487^{\mathrm{a}-\mathrm{d}}$ \\
\hline G10 & Holker & & $86^{\text {cde }}$ & $141^{\mathrm{f}-\mathrm{k}}$ & $106^{b-f}$ & $48^{\mathrm{f}-\mathrm{j}}$ & $41^{\mathrm{ab}}$ & $47.5^{\mathrm{e}-\mathrm{h}}$ & $69.6^{\mathrm{abc}}$ & $4043^{\mathrm{bcd}}$ \\
\hline G11 & HB $1963 *$ & & $87^{\mathrm{cd}}$ & $145^{\mathrm{c}-\mathrm{f}}$ & $104^{\mathrm{c}-\mathrm{g}}$ & $57^{\mathrm{b}-\mathrm{h}}$ & $28^{\mathrm{b}-\mathrm{f}}$ & $50.4^{\mathrm{b}-\mathrm{e}}$ & $70.5^{\mathrm{a}}$ & $4785^{\mathrm{ab}}$ \\
\hline G12 & HB 1964 & & $81^{\mathrm{g}-\mathrm{j}}$ & $140^{\mathrm{f}-\mathrm{k}}$ & $106^{\mathrm{b}-\mathrm{e}}$ & $53^{\mathrm{d}-\mathrm{i}}$ & $30^{\mathrm{b}-\mathrm{e}}$ & $56.6^{\mathrm{a}}$ & $67.1^{\text {cde }}$ & $4409^{a-d}$ \\
\hline G13 & KWS-Dante & & $96^{\mathrm{b}}$ & $149^{\text {bcd }}$ & $55^{\mathrm{ij}}$ & $67^{\mathrm{a}-\mathrm{f}}$ & $9^{g}$ & $38.4^{\mathrm{k}}$ & $63.8^{\mathrm{fg}}$ & $1936^{\mathrm{h}}$ \\
\hline G14 & KWS-Eileen & & $95^{\mathrm{b}}$ & $152^{\mathrm{b}}$ & $60^{\mathrm{ij}}$ & $65^{\mathrm{a}-\mathrm{g}}$ & $17^{\mathrm{d}-\mathrm{g}}$ & $52.6^{\mathrm{ab}}$ & $66.9^{\mathrm{b}-\mathrm{g}}$ & $1905^{\mathrm{h}}$ \\
\hline G15 & KWS_Grinada & & $94^{\mathrm{b}}$ & $146^{\mathrm{b}-\mathrm{g}}$ & $52^{\mathrm{j}}$ & $69^{a-e}$ & $9^{g}$ & $39.0^{\mathrm{jk}}$ & $68.6^{\mathrm{a}-\mathrm{e}}$ & $2193^{\mathrm{gh}}$ \\
\hline G16 & KWS-Hobbs & & $97^{\mathrm{ab}}$ & $150^{\mathrm{bc}}$ & $60^{\mathrm{ij}}$ & $63^{\mathrm{a}-\mathrm{h}}$ & $14^{\text {efg }}$ & $38.5^{\mathrm{k}}$ & $65.5^{\mathrm{efg}}$ & $2870^{\text {fgh }}$ \\
\hline G17 & KWS-Sassy & & $102^{\mathrm{a}}$ & $164^{\mathrm{a}}$ & $63^{\mathrm{ij}}$ & $67^{\mathrm{a}-\mathrm{f}}$ & $20^{\mathrm{c}-\mathrm{g}}$ & $43.1^{\mathrm{ij}}$ & $66.0^{\mathrm{d}-\mathrm{g}}$ & $2691^{\text {gh }}$ \\
\hline G18 & KWS_Canton & & $99^{\mathrm{ab}}$ & $152^{\mathrm{b}}$ & $66^{\mathrm{i}}$ & $49^{e-j}$ & $21^{\mathrm{c}-\mathrm{g}}$ & $43.1^{\mathrm{ij}}$ & $66.7^{\mathrm{c}-\mathrm{g}}$ & $3050^{\text {efg }}$ \\
\hline G19 & KWS-Solicit & & $96^{\mathrm{b}}$ & $148^{\mathrm{b}-\mathrm{e}}$ & $63^{\mathrm{ij}}$ & $72^{\mathrm{a}-\mathrm{d}}$ & $14^{\mathrm{efg}}$ & $37.3^{\mathrm{k}}$ & $66.2^{\mathrm{d}-\mathrm{g}}$ & $2430^{\mathrm{gh}}$ \\
\hline G20 & IBON-HI13/14-41 & & $77^{\mathrm{jkl}}$ & $140^{\mathrm{f}-\mathrm{k}}$ & $88^{\mathrm{h}}$ & $66^{\mathrm{a}-\mathrm{g}}$ & $21^{\mathrm{c}-\mathrm{g}}$ & $46.8^{\mathrm{e}-\mathrm{i}}$ & $68.1^{\mathrm{a}-\mathrm{e}}$ & $3799^{\mathrm{def}}$ \\
\hline G21 & IBON-HI14/15-45 & & $77^{\mathrm{jkl}}$ & $137^{\mathrm{ijk}}$ & $100^{\mathrm{c}-\mathrm{g}}$ & $53^{b-j}$ & $23^{\mathrm{b}-\mathrm{g}}$ & $50.6^{\mathrm{b}-\mathrm{f}}$ & $69.4^{\mathrm{a}-\mathrm{d}}$ & $4300^{\mathrm{a}-\mathrm{d}}$ \\
\hline G22 & IBON-HI14/15-56 & & $82^{\mathrm{e}-\mathrm{j}}$ & $136^{\mathrm{jk}}$ & $95^{\text {gh }}$ & $80^{\mathrm{a}}$ & $23^{\mathrm{b}-\mathrm{g}}$ & $48.3^{\mathrm{b}-\mathrm{g}}$ & $66.1^{\mathrm{d}-\mathrm{g}}$ & $4791^{\mathrm{abc}}$ \\
\hline G23 & IBON-HI14/15-96 & & $81^{\mathrm{f}-\mathrm{j}}$ & $138^{\mathrm{f}-\mathrm{k}}$ & $101^{\mathrm{c}-\mathrm{g}}$ & $31^{\mathrm{j}}$ & $23^{\mathrm{b}-\mathrm{g}}$ & $47.5^{\mathrm{c}-\mathrm{i}}$ & $69.0^{\mathrm{a}-\mathrm{e}}$ & $4392^{\mathrm{a}-\mathrm{d}}$ \\
\hline G24 & IBON-HI14/15-102 & & $78^{i-1}$ & $138^{g-k}$ & $100^{\mathrm{d}-\mathrm{g}}$ & $48^{e-j}$ & $23^{\mathrm{b}-\mathrm{g}}$ & $46.4^{\mathrm{f}-\mathrm{i}}$ & $67.4^{\mathrm{a}-\mathrm{f}}$ & $4700^{\mathrm{a}-\mathrm{d}}$ \\
\hline G25 & IBON-HI14/15-147 & & $77^{\mathrm{i}-1}$ & $139^{\mathrm{f}-\mathrm{k}}$ & $96^{\mathrm{e}-\mathrm{h}}$ & $78^{\mathrm{a}}$ & $23^{\mathrm{b}-\mathrm{g}}$ & $47.0^{\mathrm{e}-\mathrm{i}}$ & $69.4^{\mathrm{a}-\mathrm{d}}$ & $4370^{a-d}$ \\
\hline G26 & MBНIBYT-23 & & $79^{h-k}$ & $141^{\mathrm{e}-\mathrm{k}}$ & $102^{\mathrm{c}-\mathrm{g}}$ & $69^{a-e}$ & $24^{\mathrm{b}-\mathrm{g}}$ & $47.7^{\mathrm{c}-\mathrm{i}}$ & $70.0^{\mathrm{abc}}$ & $4382^{\mathrm{a}-\mathrm{d}}$ \\
\hline G27 & Explorer & & $87^{\mathrm{c}-\mathrm{f}}$ & $144^{\mathrm{c}-\mathrm{i}}$ & $59^{\mathrm{ij}}$ & $74^{\mathrm{ab}}$ & $10^{\mathrm{fg}}$ & $39.5^{\mathrm{jk}}$ & $66.0^{\mathrm{d}-\mathrm{g}}$ & $2775^{\text {gh }}$ \\
\hline G28 & Traveller* & & $89^{c}$ & $145^{\mathrm{b}-\mathrm{h}}$ & $88^{\mathrm{h}}$ & $48^{e-j}$ & $51^{\mathrm{a}}$ & $46.0^{\text {ghi }}$ & $66.9^{\mathrm{b}-\mathrm{g}}$ & $4215^{\text {bcd }}$ \\
\hline
\end{tabular}

$\mathrm{DHE}=$ days to heading, $\mathrm{DMA}=$ days to maturity, $\mathrm{PLH}=$ plant height $(\mathrm{cm}), \mathrm{SC}=$ scald $(\%), \mathrm{NB}=$ net blotch $(\%)$, $\mathrm{TKW}=$ thousand kernel weight $(\mathrm{g}), \mathrm{HLW}=$ hectoliter weight $\left(\mathrm{hl} \mathrm{g} \mathrm{g}^{-1}\right), \mathrm{GYLD}=$ grain yield $\left(\mathrm{kg} \mathrm{ha}^{-1}\right)$, §these traits were not recorded at DB18, $*=$ Improved checks

Individual environment mean grain yield and malt quality parameters value of tested malt barley genotype have been presented in Table 4. HB 52 x Bahati (G5) showed higher mean grain yield values across most environments, ranged from $3614-7026 \mathrm{~kg} \mathrm{ha}^{-1}$. Besides the grain yield the genotypes had better malt quality, it recorded 80.7, 8.3, 72, 699 values for extract, protein, friability and beta-glucan respectively. Similarly, the check variety (HB1963), exhibited the next high mean grain yield at most test environment and it also showed better values in malt quality traits. This confirmed that the variety is an alternative malting barley genotype for malting industry. Then IBON 174/03 x Traveller had higher mean grain yield values across test environments, but the genotype exhibited relatively poor malting quality. On the other hand, Bekoji-1 x Grace and Sabini x Beka showed premium malt qualities, they scored 81.8 and 81.5 for extract, 10.0 and 9.8 for protein, 85 and 78 for friability and 369 and 287 for beta glucan, respectively. These traits are the most important and relevant for the malt factories and breweries so that the breeders are working on improving it. Accordingly, the G8 and G7 scored mean grain yield value ranged $2915-5090 \mathrm{~kg} \mathrm{ha}^{-1}$ and 2804-6248 $\mathrm{kg} \mathrm{ha}^{-1}$ across the test environments with the high malt quality standard. (Table 4). Moreover G3 showed better values in all malt quality parameters. However, unlike other genotypes these values were recorded using NIRs techniques. So, the values should be further confirmed using wet chemistry method for solid conclusion. Consequently, even if HB 52 x Bahati and IBON 174/03 x Traveller were higher in grain yield performance, we can recommend genotype Bekoji-1 x Grace instead for its premium malt barley quality and acceptable grain yield potential. 
Table 4. Individual location average grain yield and malt quality traits performances of the 12 malt barley genotypes

\begin{tabular}{|c|c|c|c|c|c|c|c|c|c|c|c|c|c|}
\hline Trt\# & Genotype & & $\overline{\text { BK17 }}$ & $\overline{\text { BK18 }}$ & $\overline{\text { DB18 }}$ & HO17 & KF17 & $\overline{\text { KF18 }}$ & Mean & $\begin{array}{l}\text { Extract } \\
\%\end{array}$ & $\begin{array}{l}\text { Protein } \\
\%\end{array}$ & $\begin{array}{l}\text { Friability } \\
\%\end{array}$ & $\begin{array}{l}\begin{array}{l}\text { Beta } \\
\text { glucan } \\
(\mathrm{mg} / \mathrm{l})\end{array} \\
\end{array}$ \\
\hline G1 & Grace x HB 1307 & & 3370 & 4820 & 3013 & 3611 & 4159 & 4872 & 3995 & 80.4 & 7.6 & 70 & 462 \\
\hline $\mathrm{G} 2$ & Bekoji I xBahati & & 3651 & 5813 & 2640 & 3101 & 3947 & 4822 & 3972 & $81.1^{*}$ & $13.5^{*}$ & $68^{*}$ & $333^{*}$ \\
\hline G3 & HB 1307 x Su-Lilly & & 4236 & 6396 & 3634 & 2572 & 4015 & 2629 & 3920 & $84.0^{*}$ & $9.5^{*}$ & $86^{*}$ & $246^{*}$ \\
\hline G4 & Belgium 2 & & 3873 & 5926 & 2972 & 3325 & 3784 & 4426 & 4049 & 76.8 & 13.3 & 29 & 1000 \\
\hline G5 & HB $52 \times$ Bahati & & 5304 & 7026 & 3614 & 4293 & 4675 & 6046 & 5128 & 80.7 & 8.3 & 72 & 699 \\
\hline G6 & $\begin{array}{ll}\text { IBON } & 174 / 03 \\
\text { Traveller } & \end{array}$ & $\mathrm{x}$ & 3595 & 6265 & 3154 & 4019 & 4715 & 5075 & 4470 & 76.7 & 12.2 & 37 & 1000 \\
\hline G7 & Sabini $\times$ Beka & & 4127 & 6248 & 2804 & 3441 & 3633 & 3287 & 3931 & 81.5 & 9.8 & 78 & 287 \\
\hline G8 & Bekoji-1 x Grace & & 3880 & 5090 & 2915 & 4081 & 4000 & 3968 & 4027 & 81.8 & 10.0 & 85 & 369 \\
\hline G9 & IBON 174/03 & & 3874 & 6667 & 2348 & 4236 & 4855 & 5144 & 4487 & 79.4 & 10.6 & 50 & 1000 \\
\hline G10 & Holker & & 3477 & 6373 & 3035 & 3658 & 3418 & 4179 & 4043 & 77.6 & 13 & 61 & 420 \\
\hline G11 & HB 1963 & & 4005 & 5818 & 3686 & 5355 & 5198 & 4252 & 4785 & 81.3 & 8.9 & 78 & 510 \\
\hline G12 & HB 1964 & & 3645 & 5350 & 2927 & 4277 & 4584 & 5625 & 4409 & 78.6 & 11.6 & 44 & 1000 \\
\hline
\end{tabular}

*These data were recorded from Near infrared spectroscopy (NIRs) using Bruker Tango instrument $\mathrm{NB}=$ malt quality standards: Extract, $>78 \%$, Friability, $>77 \%$, Beta glucan, $<400 \mathrm{mg} / \mathrm{l}$, Protein, $9-11.5$

\section{GGE BIPLOT}

Significant mean squares for G X E indicated inconsistency of mean grain yield performance of genotypes in different environments. Studying the causes of G X E interaction helps to establish breeding objectives and identify areas of optimal cultivar adaptation (Yan and Hunt, 2001). The "which-won-where" view of the GGE biplot is important feature for mega environment identification. Therefore in this study based on the mean yield performance of malt genotypes, all environments fall into single sector. The six environments (KF17, KF18, BK17, BK18, DB18 and HO17) grouped as one mega environment and G5, G6, G9 and G11 performed well in all environments included in mega environments (Figure 1). In addition, G5 (HB 52 x Bahati) is the vertex genotype, which showed the superiority of the genotype in mean grain yield performance than the other genotypes included in the mega environment (Yan and Tinker, 2006). Moreover, no environments fell into sectors that contained the remaining genotypes, which indicates that they were the poorest genotypes in all test environments (Yan, 2001).

\section{Which Won Where/What}

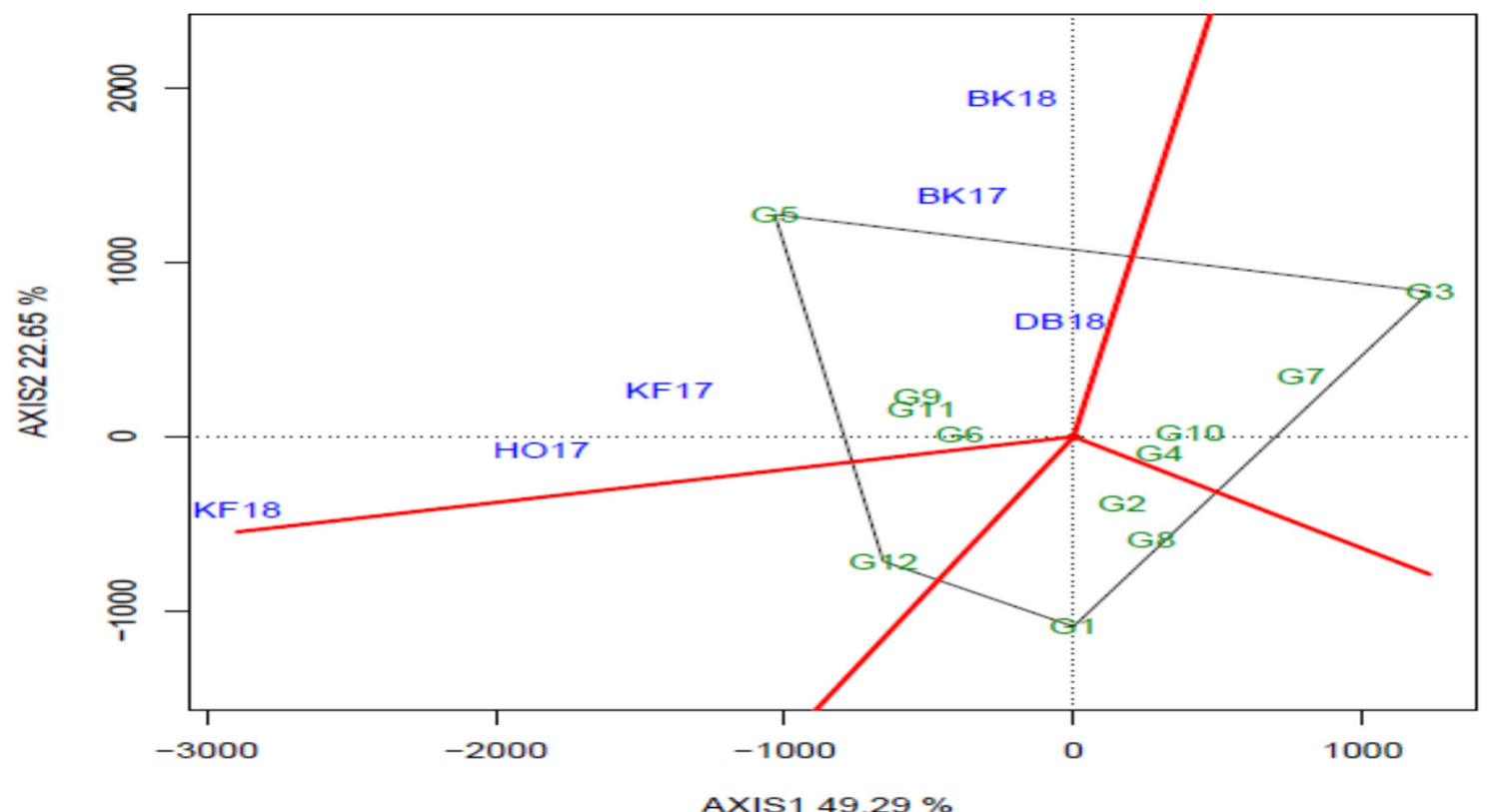

Figure 1. The which-won-where view of the GGE biplot of grain yield of Food barley genotypes based on the G $\times$ E data

The GGE biplot explained $72 \%$ of the grain yield variation due to GGE (Figure 2). "Mean vs. Stability" view of GGE biplot is efficient tool to compare genotype based on mean performance and stability across environments within a mega-environment (Yan et al., 2007). Mean vs. stability view of GGE biplot presented in Figure 2. G-5 showed higher mean grain yield value than the other test genotypes and had moderate stability. The check varieties G-9 (IBON 174/03), G-11 (HB 1963) and the other test genotype G-6 (IBON 174/03 x Traveller) had the next highest mean grain values and these genotypes revealed good stability. On the other hand among high malt quality 
yielding genotypes, G8 (Bekoji-1 x Grace) showed relatively better stability (Figure 2).

\section{Mean vs. Stability}

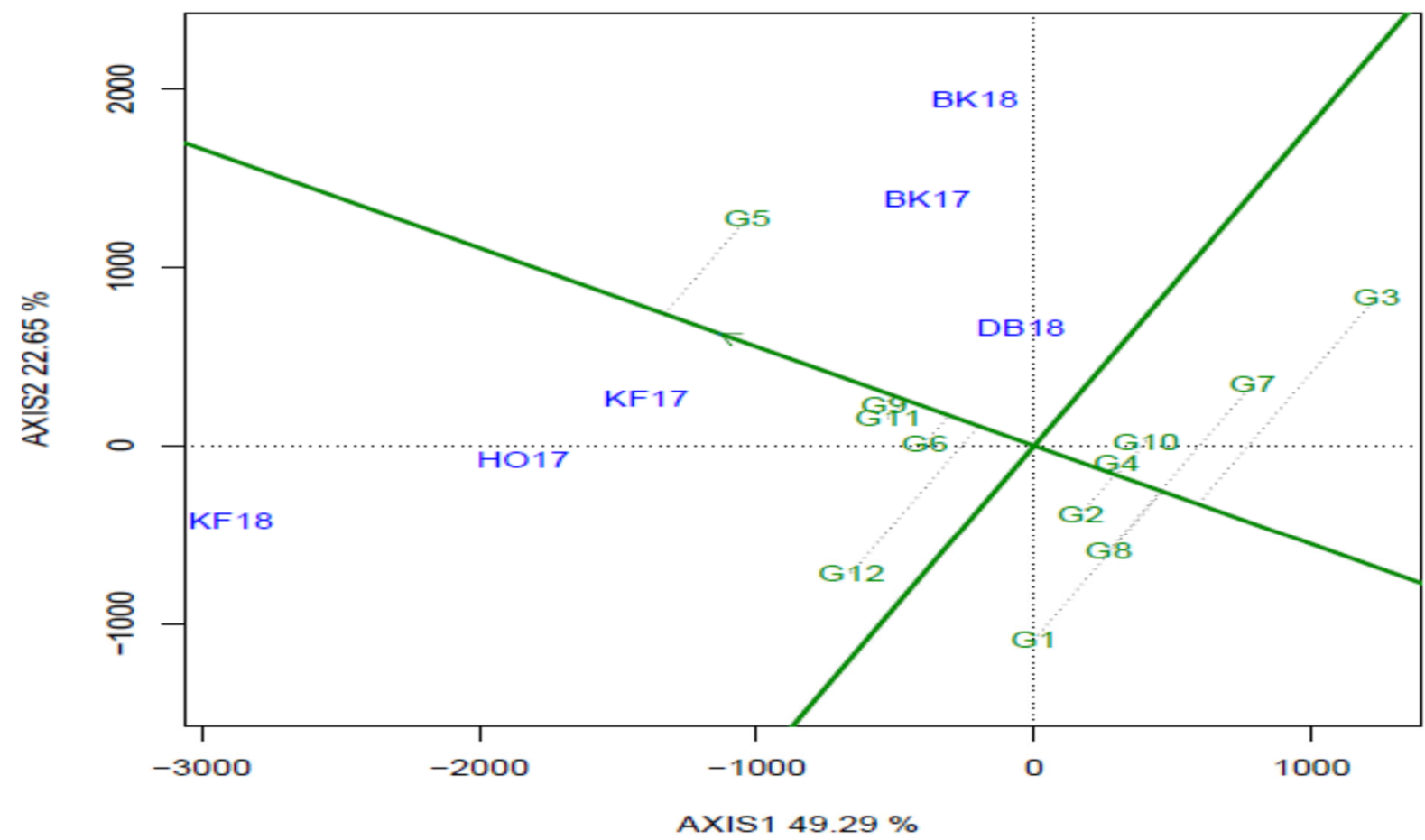

Figure 2. Mean grain yield performance and stability of genotypes based on the $\mathrm{G} \times \mathrm{E}$ data

\section{CONCLUSIONS}

The major finding from this study was that G-8 (Bekoji-1 x Grace) had shown a premium malt and physical grain quality result meeting the standard of the malt industry, which in most cases are missing in our elite varieties. The other genotype G-5 (HB 52 x Bahati) showed significantly higher mean grain yield and good malt quality traits, except for friability. The GGE biplot, G5 (HB 52 x Bahati) was the winning genotype in all test environments. Moreover, the "mean vs stability" view of GGE biplot indicated that G-5 recorded the highest mean grain yield. In terms of stability, the high yielding genotype (G-5) and the high quality genotype (G-8) showed moderate stability across test environments. Overall, genotypes G-8 is identified as potential malt barley candidate variety for further malt quality test prior to variety verification trial. Genotype G-5 is included in the crossing block as potential donor parent for its high yielding performance across the test environments. In addition, G-8 (Bekoji-1 $\mathrm{x}$ Grace) and G-7 (Sabini x Beka) are recommended for the malt barley crossing program as potential parent for their good malt quality traits (high malt extract and low beta-glucan).

\section{REFERENCES}

Bayeh, M and Berhane L. 2011. Barley research and development in Ethiopia - an overview. 1n: Mulatu, B. and Grando, S. (eds). 2011. Barley Research and Development in Ethiopia. Proceedings of the 2nd National Barley Research and Development Review Workshop. 28-30 November 2006, HARC, Holetta, Ethiopia. ICARDA, PO Box 5466, Aleppo, Syria. pp xiv + 391

Berhane Lakew, Chilot Yirga and Wondimu Fikadu, 2016. Malt Barley Research and Development in Ethiopia : Challenges and Opportunities. In: Dawit Alemu, Eshetu Derso, Getnet Assefa and Abebe Kirub (eds.). Agricultural Research for Ethiopian Renascence: Challenges and Opportunities. Proceedings of the national conference on Agricultural Research for Ethiopian Renascence. 26-17 January 2016, UNECA, Addis Abeba.

CSA, 2018. Area and Production of Major Crops, Agricultural Sample Survey, 2017/18 (2010E.C.). Central statistics Agency, Statistical Bulletin 586. Addis Abeba, Ethiopia

Ethiopian Revenues and Customs Authority - ERCA, 2017 : http://www.erca.gov.et.

European Brewery Convention, 1998. Analytica EBC. 5th ed. Verlag Hans Carl, Nurnberg, Germany.

R Core Team, 2017. R: A language and environment for statistical computing. R Foundation for Statistical Computing, Vienna, Austria. URL http://www.R-project.org/.

Singh, M. and Ceccarelli, S., 1996. Estimation of heritability of crop traits from variety trial data. Technical Manual No. 21. International Center for Agricultural Research in the Dry Areas, Aleppo, Syria.

Yan, W., 2001. GGEbiplot-A Windows application for graphical analysis of multienvironment trial data and 
other types of two-way data. Agronomy journal, 93(5), pp.1111-1118.

Yan, W. and Tinker, N.A., 2006. Biplot analysis of multi-environment trial data: Principles and applications. Canadian journal of plant science, 86(3), pp.623-645.

Yan, W., Kang, M.S., Ma, B., Woods, S. and Cornelius, P.L., 2007. GGE biplot vs. AMMI analysis of genotypeby-environment data. Crop science, 47(2), pp.643-653. 